\title{
Élaboration et évolution du discours touristique sur un espace rural : l'exemple du Marais breton
}

The Formulation and Evolution of Tourism Discourse in a Rural Area: The Example of the Breton Marsh

\section{Johan Vincent}

\section{(2) OpenEdition}

\section{Journals}

Édition électronique

URL : http://journals.openedition.org/abpo/2879

DOI : $10.4000 / a b p o .2879$

ISBN : 978-2-7535-3977-8

ISSN : 2108-6443

\section{Éditeur}

Presses universitaires de Rennes

\section{Édition imprimée}

Date de publication : 15 décembre 2014

Pagination : 157-176

ISBN : 978-2-7535-3975-4

ISSN : 0399-0826

Référence électronique

Johan Vincent, «Élaboration et évolution du discours touristique sur un espace rural : I'exemple du Marais breton », Annales de Bretagne et des Pays de l'Ouest [En ligne], 121-4 | 2014, mis en ligne le 15 décembre 2016, consulté le 19 avril 2019. URL : http://journals.openedition.org/abpo/2879; DOI : $10.4000 / a b p o .2879$ 


\title{
Élaboration et évolution du discours touristique sur un espace rural : l'exemple du Marais breton
}

\author{
Johan VINCENT \\ chercheur associé au CERHIO (UMR 6258)
}

Dans un article précédent ${ }^{1}$, je m'étais intéressé au rôle des guides touristiques dans la communication des stations balnéaires, promptes à créer des lieux idéaux, en renforçant les atouts des destinations - encouragés ainsi à être mieux mis en valeur localement - et, éventuellement, en réduisant l'impact de leurs défauts. Mais comment ce rôle de garant ${ }^{2}$ se traduit-il sur un espace qui se trouve aux abords d'une région touristique, sans être, à l'origine du moins, touristique lui-même? Étudions-le à partir d'un cas particulier : le Marais breton. Cet espace géographique se situe au bord de l'océan Atlantique, à la frontière des départements de la Loire-Atlantique et de la Vendée. Doté d'un réseau de canaux et de prairies humides, il a été constitué à partir du xi ${ }^{\mathrm{e}}$ siècle par poldérisation sur la baie de Bourgneuf et sa superficie atteint aujourd'hui environ $450 \mathrm{~km}^{2}$.

Pour comprendre le discours touristique sur le Marais breton, un corpus de 48 guides locaux ou régionaux grand public a été établi, dont une partie a été rassemblée au cours d'une mission destinée à constituer le cahier de contenu sur le tourisme, effectuée pour le programme de recherche " Muséographie 2013-2016 " de l'écomusée du nord-ouest vendéen, Le Daviaud. Le plus ancien guide évoquant la région est imprimé en $1829^{3}$, le plus récent date de $2013^{4}$. Chaque texte a été recopié au mot près (environ 378000 caractères espaces compris) mais les images n'ont pas

1. VINCENT, Johan, « Les paradis de papier : les sites balnéaires bretons magnifiés par les guides (1860-1939) ", Annales de Bretagne et des pays de l'Ouest, tome 119, 2012, n 4, p. 99-113.

2. SECANE, Annabelle, Les mécanismes énonciatifs dans les guides touristiques, entre genre et positionnements discursifs, Paris, L'Harmattan, 2013, p. 51.

3. Genoude, Eugène, Voyage dans la Vendée et dans le Midi de la France, Paris/Lyon, Méquignon Fils aîné/Périsse Frères, 1829, 420 p.

4. Guide BaLaDO, Pays de la Loire, Paris, Éditions Mondéos, 2013, 476 p. 
fait l'objet d'une étude précise. Notons tout de suite que le corpus présente une faiblesse puisque, avec trois exemplaires, les années 1980 sont sousreprésentées. Ceci s'explique par plusieurs raisons : les bibliothèques des communes du Marais breton renouvellent régulièrement leur collection de guides sans constituer de fonds anciens, vendant les anciens exemplaires dans des opérations de déstockage; la bibliothèque du Touring-Club de France cesse ses activités en 1983 et, bien qu'elle soit encore alimentée, elle a sans doute été moins bien approvisionnée le temps de son transfert à la Ville de Paris; les centres des archives départementales ne paraissent pas avoir reçu ou acheté d'exemplaires de guide, sauf ceux d'organismes départementaux (comme les comités départementaux de tourisme); les centres des archives municipales, quand ils existent et quand leurs fonds sont classés, possèdent des versements anciens mais pas toujours les archives des syndicats d'initiatives alors constitués sous des formes distinctes des services municipaux (ex. : SIVOM, SEM), encore en activité.

Le discours touristique portant sur le Marais breton au cours des deux derniers siècles se forge alors que deux évolutions se développent : la reconnaissance locale du territoire, teintée progressivement de patrimonialisation, et son appropriation touristique. Quels sont les croisements qui s'opèrent dans l'élaboration des thématiques? Cet article a été réalisé en s'appuyant partiellement sur une analyse de l'ensemble du contenu de ces guides, en utilisant le logiciel de traitement quantitatif de données Tropes (version 840), développé par Pierre Molette et Agnès Landré, sur la base des travaux de Rodolphe Ghiglione (http://www.tropes.fr). Il ne fait pas une analyse linguistique de l'objet étudié et nous ne nous attarderons pas non plus sur l'interaction des collections avec le lecteur ${ }^{5}$, nous préférerons une analyse globale. Dans un premier temps, nous allons voir comment est caractérisé le Marais breton, puis nous analyserons le discours touristique selon trois périodes pour enfin nous attarder sur la dernière phase chronologique, quand patrimonialisation et touristification de l'espace se développent.

\section{Caractéristiques du Marais breton dans les guides}

\section{Un site à dimensions variables}

L'identification du Marais breton a été progressive. Tout d'abord, cet espace s'est étendu, par endiguements successifs, jusque dans les années 1960. Cet espace, appelé Marais breton, marais du Nord ou marais de Challans, afin de le distinguer du Marais poitevin, dit aussi marais du Sud ou marais de Luçon, est décrit comme une vaste plaine basse, unie, humide et nue, conquise sur la baie de Bourgneuf par les atterrissements de la mer qui décante ses vases à l'abri de la digue naturelle formée par l'île de

5. Francon, Marc, Le Guide Vert Michelin. L'invention du tourisme culturel populaire, Paris, Éditions Economica, coll. "Économies et sociétés contemporaires ", 2001. 
Noirmoutier et par l'exhaussement du $\mathrm{sol}^{6}$. Les guides du XIX ${ }^{\mathrm{e}}$ siècle prévoyaient un endiguement complet de la baie de Bourgneuf, reliant l'île de Noirmoutier au continent, avant la fin du xx siècle selon certains, ce qui faillit bien advenir ${ }^{7}$. En 1927, le guide Bleu rappelle que depuis un siècle, le territoire de Bourgneuf-en-Retz aurait gagné plus de 500 hectares $^{8}$. La notion d'" île " est fréquemment évoquée : Bouin était ainsi une île au $\mathrm{XIX}^{\mathrm{e}}$ siècle et ce souvenir perdure dans les discours jusqu'à nos jours. Sa superficie varie d'un guide à l'autre, de 20000 à 50000 hectares, d'ailleurs indépendamment des endiguements.

Ensuite, le Marais breton se trouve sur les marches historiques de la Bretagne et du Poitou. Les guides ne manquent pas de souligner cette caractéristique, en particulier pour décrire la ville de Bouin où siégeaient le sénéchal du Poitou et le sénéchal de Bretagne. Quatre communes du marais font désormais partie de la Loire-Atlantique tandis que dix-huit communes (en englobant des communes comme Saint-Gilles-Croix-de-Vie et Commequiers, qui sont situées à la frontière sud de cet espace) se trouvent en Vendée. La limite départementale va cependant scinder le Marais breton, en distinguant les communes vendéennes des communes de Loire-Atlantique, généralement marginalisées. Le nom même de Marais Breton-Vendéen que tente d'imposer le conseil général de la Vendée est révélateur de cette marginalisation souhaitée par certains, bien que la première mention de ce nouveau nom dans les guides soit finalement tardif (Guide du Routard sur les Pays-de-la-Loire de 2007). Bizarrement, les guides de Loire-Atlantique ont même eu le réflexe d'englober dans leur propos l'île de Noirmoutier et le passage du Gois (situés en Vendée), plutôt que le Marais breton, même si cette île était facilement accessible par bateau via Pornic.

Enfin, les limites du Marais breton sont difficiles à identifier parce qu'il n'existe pas de centralité. Ainsi, dans l'édition de 1897 comme dans celle de 1912, quand le Bædeker décrit en une dizaine de lignes le Marais poitevin, le Marais breton n'a droit à aucun paragraphe distinctif; seules les communes qui le composent sont évoquées. De plus, si les auteurs utilisent comme synonyme l'expression " marais de Challans ", il faut savoir que le bourg de Challans ne se trouve pas dans le marais mais au bord du marais, à la limite avec le bocage. Il n'existe aucune grande ville dans le marais. Les subtilités ajoutées sèment plutôt la confusion. L'auteur du Guide pittoresque du voyageur en France : département de la Vendée, vers 1850, divise le territoire en trois parties : marais desséché, marais mouillé et marais salants, mais son propos se situe à l'échelle départementale, sans lien avec le marais Breton ${ }^{9}$. L'auteur du guide Bleu Pays de la Loire de 1995, quant à lui, délimite arbi-

6. De la Loire aux Pyrénées, Paris, Hachette et C Cie, coll. " Guides Joanne ", 1911, p. 3.

7. VINCENT, Johan, Le Passage du Gois menacé. Un grand projet d'endiguement de la baie de Bourgneuf sous la Seconde Guerre mondiale, Nantes/Laval, Siloë, 2011, p. 13-29.

8. MonmarchÉ, Georges, Bords de la Loire et Sud-Ouest, Paris, Hachette, coll. « Guides bleus ", 1927, p. 306.

9. Guide pittoresque du voyageur en France : département de la Vendée, Nantes, Imprimerie de Firmin Didot Frères, sans date [vers 1850], p. 2. 


\section{Le Marais breton au début du $\mathrm{XXI}^{e}$ siècle}

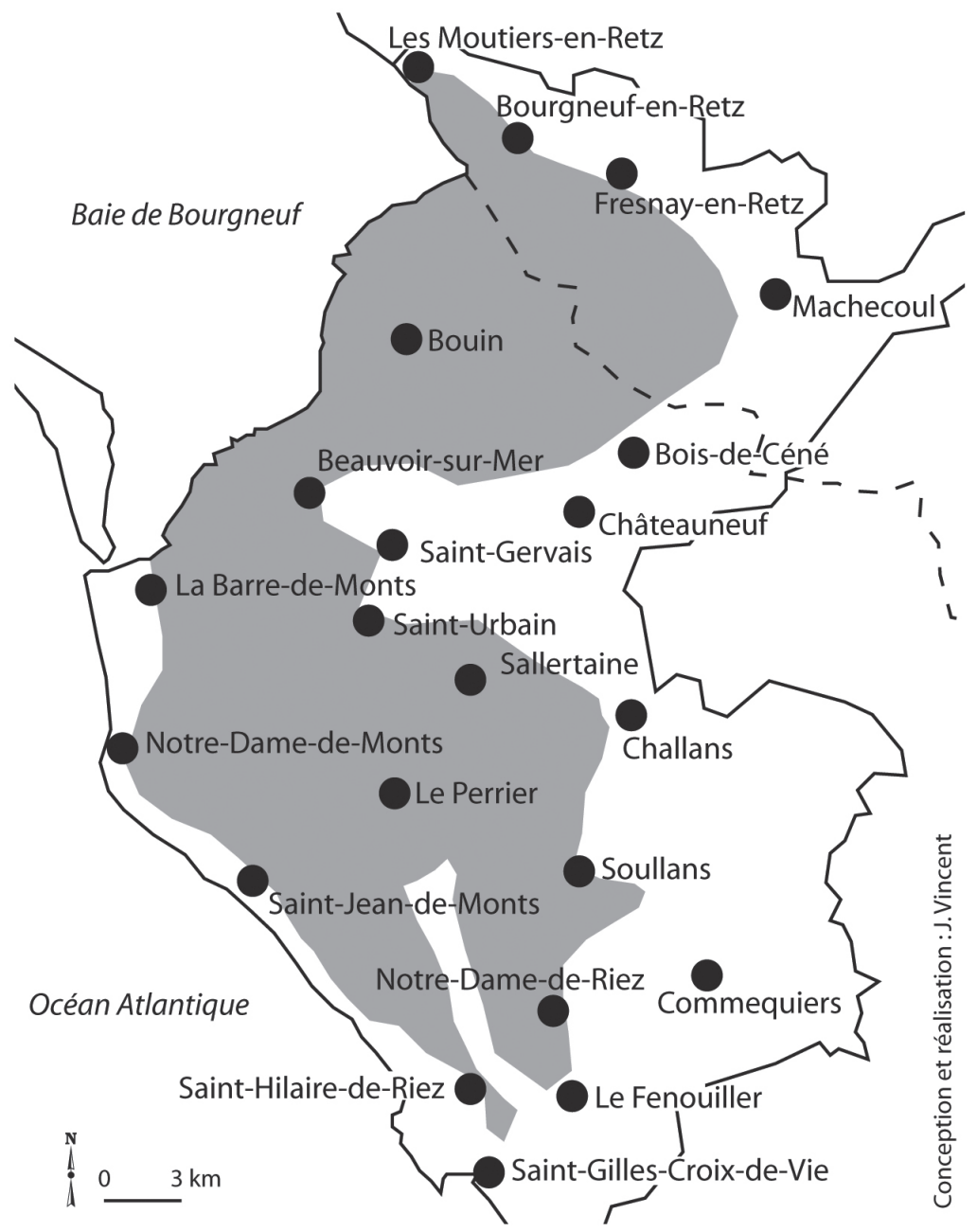

\footnotetext{
$\square$ Marais doux et marais salé

- - - - Limite départementale

Limites communales
} 
trairement les secteurs : marais de Bouin ou marais Nord d'un côté, marais de Monts de l'autre. Le marais de Monts serait plus dévolu à l'élevage ${ }^{10}$. Dans la réalité, les seules limites pertinentes à l'intérieur du Marais breton sont celles entre le marais doux et le marais salé, mais elles sont quasiment inconnues des auteurs des guides, à l'exception de deux auteurs dont les propos, rattachés au musée du Daviaud, sont très vagues ${ }^{11}$. Illes et espaces dunaires anciens disparaissent dans les descriptions générales. L'espace du Marais breton peut donc s'avérer complexe et difficile à appréhender touristiquement. C'est une complication que l'on peut retrouver pour d'autres territoires ruraux, comme la Brenne, dans l'Indre ${ }^{12}$.

\section{Un espace terrestre dominé par l'eau}

Afin de faciliter la reconnaissance touristique du milieu, les auteurs des guides choisissent d'identifier le marais Breton comme un espace où l'eau est omniprésente.

"Ses plats paysages [sont] sillonnés d'étiers et de canaux, à la poésie si particulière. Leur forme même dit le long travail de l'homme pour la conquête sur la mer. Rien n'arrête la lumière qui rase ces basses terres aux contours seulement soulignés par la ligne sombre des paysages plus élevés qui l'entourent. Le marais blanc de l'hiver, couvert d'eaux stagnantes, verdit au printemps en un immense tapis d'herbe, puis se change l'été en un paillasson jaunissant à la sécheresse ${ }^{13}$."

En termes de paysages, ce triptyque temporel reste néanmoins tardif, puisque la première mention du marais blanc dans un guide date de 1971 et se rapporte à un film, Mon marais blanc, projeté au palais des congrès de Saint-Jean-de-Monts ${ }^{14}$. Le marais blanc désigne le marais entièrement inondé qui, sous un ciel gris, prend cette couleur. Alors qu'on peut considérer que le marais n'a plus jamais été blanc depuis 1987, cette mention est systématiquement reprise à partir des années 1990. Par contre, les auteurs des guides précédents ont de nombreux moyens de faire savoir que l'eau est omniprésente en hiver, en relation avec le mode de déplacement des maraîchins : la yole, ou niole, une barque à fond plat, et Eugène Genoude constate dès 1829 que " le Marais est couvert de barques dans l'hiver ${ }^{15}$ ".

10. Guide Bleu, Pays de la Loire, Paris, Hachette, 1995 [1 ${ }^{\text {re }}$ éd. 1989], p. 216-225.

11. BÉly, Gilles et Bouflet, Bertrand, Vendée, tourisme et culture, La Roche-sur-Yon, Siloë éditeur, coll. " Guides Siloë ", 2001, p. 218, et Guide du Routard, Pays de la Loire, Paris, Hachette, 2007, p. 450.

12. Blouin-GouRBILIÈRE, Claire, L'élaboration d'images "paysages " habitantes : un levier participatif d'aménagement du territoire. Le cas du Parc naturel régional de la Brenne (Indre, France), Thèse de doctorat en géographie et aménagement de l'espace, Université d'Angers-Agrocampus Ouest, 2013, p. 98 et p. 110.

13. Guide Bleu, Pays de la Loire, op. cit., p. 216.

14. Saint-Jean-de-Monts, Guide officiel du Syndicat d'Initiatives Office du Tourisme, SaintJean-de-Monts, 1971, p. 55.

15. Genoude, Eugène, Voyage dans la Vendée..., op. cit., p. 105. 
Le logiciel Tropes désigne une prédominance des thématiques relatives à l'eau dans les deux univers de référence ${ }^{16}$. Dans le contexte général, sur l'ensemble des guides, les mots " mer " ( $2^{\mathrm{e}}$ rang, 901 occurrences), " eau " ( $3^{\mathrm{e}}$ rang, 853 occurrences), " marine " (11 $\mathrm{e}$ rang, 322 occurrences) sont très présents. Dans le contexte détaillé, les mots ou expressions " cours d'eau " ( $2^{\mathrm{e}}$ rang, 721 occurrences), " mer " ( $3^{\mathrm{e}}$ rang, 694 occurrences), " île " (13 $\mathrm{rang}$, 195 occurrences), voire " port " ( $17^{\mathrm{e}}$ rang, 152 occurrences) se rapportent à l'univers aquatique. Le premier rang est à chaque fois occupé par une référence générale liée aux noms de lieu (France et Pays-de-la-Loire).

Seule survivance de cette omniprésence de l'eau, l'étier demeure un élément important. Le mot est utilisé dans de nombreux guides depuis le milieu du XIX ${ }^{\mathrm{e}}$ siècle. Dans le Marais breton, il a un sens plus large que celui d'un canal conduisant l'eau de mer dans les marais salants : il sert à drainer les terres du marais en conduisant l'eau recueillie vers la mer et, pour le cas du marais salé, en amenant au moment de la marée haute l'eau de mer vers l'intérieur du marais. S'il est absent, c'est le mot " canaux " qui le remplace, avec une moindre utilisation depuis le milieu des années 1990. Les deux termes sont parfois associés. Au cours de la visite, cette caractéristique permet une reconnaissance immédiate du Marais breton par rapport aux autres territoires environnants : s'il est difficile à appréhender à la petite échelle (aspect global), il est de suite identifié à la grande échelle (aspect local).

\section{Des lieux marqués par la religion et la tradition?}

Avec 538 occurrences dans le contexte général ( $6^{\mathrm{e}}$ rang), 314 dans le contexte détaillé ( $7^{\mathrm{e}}$ rang, à quoi se rajoutent les 195 occurrences de " christianisme " et son $12^{\mathrm{e}}$ rang), la place de religion dans les guides ne peut être ignorée. Mais cette thématique n'est pas facile à aborder car elle recouvre plusieurs sujets : les bâtiments religieux, la pratique religieuse, l'histoire de la Vendée durant la Révolution française.

La mention de la religion est moins marquée dans les guides d'avant la Première Guerre mondiale. Cette faiblesse est due à l'absence d'intérêt pour les églises locales. Les églises sont pour la première fois mentionnées dans le guide d'Adolphe Joanne de 1886 sur la Vendée, dans le descriptif alphabétique de chaque commune : celles de Beauvoir-sur-Mer, de l'ÎleChauvet à Bois-de-Céné, de Bouin, de Notre-Dame-de-Monts, de Saint-Gillessur-Vie, de Sallertaine, de la Lande-en-Beauchêne à Sallertaine, dont deux à l'état de ruines ${ }^{17}$. En Loire-Atlantique, seule l'église de Bourgneuf-en-Retz,

16. Le dictionnaire des équivalents sémantiques du logiciel Tropes est construit sur trois niveaux de classification. Au niveau le plus bas se situent les références utilisées, elles-mêmes regroupées de façon plus large dans les univers de référence 2 (contexte détaillé), qui à leur tour sont regroupés dans les univers de référence 1 (contexte général).

17. JoAnne, Adolphe, Géographie du département de la Vendée, Paris, Hachette et Cie, 1886. 
" belle église ogivale moderne ", retient l'attention de l'auteur ${ }^{18}$. Sans les guides Joanne, nous n'aurions d'ailleurs quasiment aucune mention des églises au XIX ${ }^{\mathrm{e}}$ siècle, puisque seul Ardouin-Dumazet évoque une église, celle de Beauvoir-sur-Mer, "dont la flèche s'aperçoit de si loin, [et qui] n'a aucun caractère ${ }^{19}$ ". À partir de 1910, la mention des églises va devenir plus systématique. Au xx ${ }^{\mathrm{e}}$ siècle sont mentionnées principalement les églises de Saint-Martin à Sallertaine, de Notre-Dame à Bouin, de Saint-Philbert à Beauvoir-sur-Mer et l'abbaye Notre-Dame de l'Île-Chauvet. Formulons comme hypothèse que l'on retrouverait la même évolution dans tout discours touristique sur un territoire rural français, mais cela reste à démontrer. Quant à la pratique religieuse, corollaire potentiel à l'usage des églises, elle n'apparaît que dans un seul guide, en 1970, et dans la réédition de ce guide en 1974, pour décrire le maraîchin ${ }^{20}$. C'est peu.

Le désintérêt pour les bâtiments religieux peut paraître surprenant quand on sait que les premiers grands endiguements ont été effectués par les moines, en particulier ceux de l'abbaye de l'Île-Chauvet. L'histoire de l'action des moines n'est évoquée qu'à partir du milieu du Xx ${ }^{\mathrm{e}}$ siècle $^{21}$ et l'abbaye de l'Île-Chauvet n'est auparavant citée que pour ses "restes " ou " ruines ", sans que son histoire soit développée. Nous avons en fait un basculement de l'intérêt touristique, de la géologie, très présente dans les premiers guides comme le montre le logiciel Tropes (sous le vocable " minéral ", au $11^{\mathrm{e}}$ rang du contexte général pour les guides avant $1914,12^{\mathrm{e}}$ rang pour les guides entre 1914 et 1960 et au $26^{\mathrm{e}}$ rang pour les guides après 1960 , enfin $30^{\mathrm{e}}$ rang après 1990), à l'histoire de la constitution du marais. Il s'agit là d'une adaptation du contenu des guides aux appétences du public, peu familier dorénavant avec la géologie mais plus séduit par le génie humain.

Le Marais breton est également rattaché à quelques épisodes de la Révolution française dans les guides touristiques mais les mentions sont finalement rares. Eugène Genoude explique en 1829 que, cerné par quarante mille hommes, il est resté inexpugnable grâce à la facilité de lâcher les écluses et d'inonder le pays ${ }^{22}$; un parti pris idéologique puisque les armées républicaines ont meurtri plusieurs communes de la région. Plus souvent est remémoré le mouvement insurrectionnel qui débute par les massacres de Machecoul en mars $1793^{23}$, qui se poursuit par la prise de Challans sous l'autorité de François Athanase Charette de La Contrie que les hommes étaient allés chercher dans son manoir de Fonteclose, à La Garnache (9 kilomètres au sud de Machecoul, 5 kilomètres au nord de Challans). Sont éga-

18. JoAnne, Adolphe, Géographie de la Loire-Inférieure, Paris, Hachette et Cie, 6 e éd., 1894.

19. ARdouin-DumAZet, Voyage en France : $3^{e}$ série, les îles de l'Atlantique, Paris/Nancy, Berger-Levrault et Cie, 1895, p. 205.

20. Comité départemental du Tourisme, La Vendée, guide officiel, Toulouse, Éditions Larrieu-Bonnel, 1970, p. 29.

21. L'Opinion économique et financière, édition illustrée. T. IV Le Pays nantais et la Vendée, $\mathrm{n}^{\circ} 7$, mars 1951, p. 82.

22. Genoude, Eugène, Voyage dans la Vendée..., op. cit., p. 105.

23. MonmarchÉ, Georges, Bords de la Loire..., op. cit., 1927, p. 304. 
lement rappelés les derniers jours de la première campagne de l'armée de Charette, quand l'armée républicaine entre le 6 décembre 1793 dans la ville de Bouin, déserte, car les hommes avaient fui par les canaux et les étiers tandis que les femmes s'étaient cachées dans le clocher de l'église ${ }^{24}$. La reconstruction de cette église en 1875 n'a pas tout à fait coupé le lien avec cette histoire. La tentative de soulèvement de la Vendée par Louis de La Rochejaquelin en 1815 est également mentionnée à plusieurs reprises, surtout avant la Première Guerre mondiale. Cet épisode est à chaque fois mis en relation avec la stèle du lieu-dit des Mathes, là où il fut abattu, même s'il y a deux mentions du lieu de son enterrement provisoire au Perrier ${ }^{25}$. À part chez Eugène Genoude, les points de vue idéologiques de la population locale sont toujours passés sous silence. L'historien Alain Gérard rappelle à ce propos que la théorie d'un peuple fidèle à sa foi est sans doute une construction mémorielle récente ${ }^{26}$, ce qui expliquerait le peu de place accordée à cette question dans les guides. L'histoire révolutionnaire est peu mise en avant localement, comme le prouve une longue absence d'entretien du monument des Mathes, jusqu'en 1996, ou le délitage de l'intéressant monument funéraire de François Crochet, dans les bras duquel La Rochejaquelein est mort. De plus, le Marais breton a une position à part dans l'histoire des guerres de Vendée mais cet aspect est complètement occulté.

\section{Une élaboration touristique progressive}

\section{Avant 1914 : découverte éthérée et inquiétante du Marais breton}

Dans un premier temps, le discours touristique se réfère à des généralités sur le Marais. L'appréhension apparaît neutre chez certains. Eugène Genoude compare le Marais, menacé sans cesse par la mer, aux plaines de la Lombardie ${ }^{27}$. Pour d'autres, l'aspect malsain du site en déconseille le séjour. La ville de Bourgneuf-en-Retz apparaît ainsi comme "bâtie sur un terrain bas et marécageux, près d'anciens marais en partie desséchés qui en rendent le séjour malsain ${ }^{28}$ ". L'auteur du guide sur la Vendée, dans la même collection, est plus disert sur les marais : marais desséché, marais mouillé, marais salants mais il signale que " l'air du marais est en général chargé de vapeurs humides et malsaines qui s'élèvent des fossés et des canaux, et qui entraînent avec elles les émanations délétères des plantes,

24. Guides Gallimard, La Vendée maritime, Paris, Éditions Nouveaux-Loisirs, 1994, p. 143144, et BÉLY, Gilles, et Bouflet, Bertrand, op. cit., p. 192.

25. Dans Genoude Eugène, et dans le Midi de la France, Paris/Lyon, Méquignon Fils aîné/Périsse Frères, 1829 et dans Guides Gallimard, La Vendée maritime, Paris, Éditions Nouveaux-Loisirs, 1994.

26. GERARD, Alain, La guerre de Vendée, La Roche-sur-Yon, Centre vendéen de recherches historiques, coll. "La Vendée, les indispensables ", 2006, p. 67.

27. GEnoude, Eugène, Voyage dans la Vendée..., op. cit., p. 105.

28. Guide pittoresque du voyageur en France : département de la Loire-Inférieure, Nantes, Imprimerie de Firmin Didot Frères, sans date [vers 1850], p. 18. 
des insectes et des reptiles qui périssent et se décomposent dans les eaux $^{29}$ ". Cette critique perdure longtemps, puisqu'on la retrouve dans le guide Joanne de 1894. Le discours change au tournant des XIX ${ }^{\mathrm{e}}$ et XX $\mathrm{X}^{\mathrm{e}}$ siècles. Le guide Les petits trous pas chers rassure les touristes qui souhaitent aller dans la station balnéaire de Saint-Jean-de-Monts : " au nord et au nord-est, des marais l'entourent, mais ces marais n'ont rien de pernicieux et il faut se garder de s'effrayer du mot. On y pêche quantité d'anguilles ${ }^{30}$ ". Le Marais devient un lieu de promenades :

"L'ancienne île de Bouin et quelques îlots calcaires comme celui de Sallertaine sont aujourd'hui empâtés dans cette plaine aux belles prairies sillonnées de canaux à marée, ou "étiers ", et de rigoles que les Maraîchins parcourent sur de petits bateaux plats et sautent adroitement à l'aide d'une perche ou "tringle" [sic : mauvaise compréhension de l'auteur, il s'agit de la ningle]. L'ancien rivage se reconnaît partout autour du Marais à son aspect verdoyant et ses haies vives qui contrastent avec la nudité des terres nouvelles ${ }^{31}$."

Les hommes sont clairement individualisés en termes de race. Adolphe Joanne relaie, dans ses éditions de 1886, de 1896, de 1900, l'idée que cette terre plate est habitée par une race qui, dit-on, ressemble assez peu aux hommes du bocage vendéen ${ }^{32}$. Leurs attributs sont néanmoins idéologisés. Pour le royaliste Eugène Genoude, en 1829, " les habitants du Marais sont très forts. C'est une race d'hommes particulière. Le Marais peut fournir dix mille hommes à la cause royale ${ }^{33}$ ". Pour l'auteur du Guide pittoresque du voyageur en France : département de la Vendée, vers 1850, l'habitant du marais n'est en général ni aussi fort, ni aussi vigoureux que celui du bocage : en fait, la proximité de l'eau et de l'air du marais altère sa santé. Il décrit un être rousseauiste sans le savoir :

"À l'exception [des gros propriétaires], que leur commerce oblige à de fréquents déplacements, les habitants du marais, privés de toutes communications avec les villes, sont généralement grossiers, incivils, et passent pour n'avoir qu'une intelligence médiocre. Leur vie doit paraître triste et misérable : cependant, ces digues isolées, ces demeures presque cachées sous les eaux, renferment une population heureuse de son sort. [...] Point de procès, point d'ambition, point d'orgueil, point d'attache trop vive aux biens de la terre; son seul désir, c'est de rendre heureux tout ce qui l'entoure. Sa paroisse et les villages voisins, voilà tout ce qu'il connaît de la France. Content de son état, il ne cherche point à en sortir; il n'a nul besoin de la protection des autorités, nulle envie d'obtenir la bienveillance du riche; il est roi dans sa cabane. [...] Le marais, les digues, les canaux et les fossés, les barques qui s'y croisent sous des berceaux de verdure, les déserts maré-

29. Guide pittoresque du voyageur en France : département de la Vendée, op. cit., p. 2-3.

30. "Les petits trous pas chers ». Guide des familles aux bains de mer, plages de la Manche et de l'Océan, Paris, La Fare éditeur, 9e éd. 1903, p. 291.

31. De la Loire aux Pyrénées, Paris, Hachette et C Cie, coll. " Guides Joanne ", 1911, p. 3.

32. JoAnne, Adolphe, Géographie du département de la Vendée, Paris, Hachette et Cie,

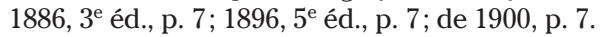

33. Genoude, Eugène, Voyage dans la Vendée..., op. cit., p. 105. 
cageux où l'on n'entend que le seul gazouillement des oiseaux, et, de loin en loin, le chant cadencé d'un yoleur, ont été son univers ${ }^{34}$."

De telles mentions sur les habitants disparaissent au début du Xx $\mathrm{x}^{\mathrm{e}}$ siècle. Quelques rares monuments historiques sont signalés. Sur les dix guides qui décrivent nommément du patrimoine, sur un total de quatorze pour la période, trois éléments sont plus fréquemment cités : le monument dédié à Louis de La Rochejaquelein, héros révolutionnaire royaliste qui avait essayé de soulever la Vendée militaire en 1815 et qui finalement mourut lors du combat des Mathes, à Saint-Hilaire-de-Riez; les traces du château démoli de Beauvoir-sur-Mer, où Henri IV survécut à une embuscade, même si l'anecdote n'est pas toujours rapportée; l'église de Beauvoir-sur-Mer, avec son curieux clocher du XII ${ }^{\mathrm{e}}$ siècle, essentiellement grâce aux guides Joanne. Leur position n'est pas hégémonique, puisqu'ils sont mentionnés la moitié du temps seulement. L'intérêt touristique de la zone apparaît donc modeste. Les autres éléments sont des églises (toujours grâce aux guides Joanne), des mégalithes, des châteaux, un fossé antique à Saint-Urbain et une tour présumée romaine à Saint-Gervais ${ }^{35}$, c'est-à-dire des monuments vraiment anciens dans un pays qui est présenté comme neuf. D'ailleurs, Léon Maître ose qualifier Bouin de site sans monument celtique, sans nom de village ancien : "Il ne pourra donc jamais se parer d'une haute antiquité ${ }^{36}$. " Le littoral commence déjà à attirer principalement les visiteurs : la première police municipale réglementant les bains à Saint-Jean-de-Monts date de $1850^{37}$, le premier établissement de bains de Saint-Gilles-sur-Vie s'installe en $1863^{38}$. L'attrait de la région n'est pas dans le Marais breton, qu'on s'empresse de traverser. En 1910, Henri Renaud enjoint néanmoins aux visiteurs d'excursionner dans les marais salants aux abords de la rivière Vie, avant qu'ils aient disparu ${ }^{39}$.

\section{De 1914 à 1960 : marginalisation face au développement des stations balnéaires voisines}

Les auteurs de guide tendant à se copier mutuellement, les caractéristiques qui émergent au début du $\mathrm{Xx}^{\mathrm{e}}$ siècle se retrouvent dans les guides de l'entre-deux-guerres mais la priorité des guides est maintenant nettement balnéaire. Le guide Bleu Bords de la Loire et Sud-Ouest, de 1927, dépeint,

34. Guide pittoresque du voyageur en France : département de la Vendée, op. cit., p. 4.

35. Cette mention d'une tour apparaît dans De la Loire aux Pyrénées, Paris, Hachette et Cie, coll. " Guides Joanne ", 1911, p. 8, et est reprise par Georges MonMARCHÉ, Bords de la Loire et Sud-Ouest, Paris, Hachette, coll. " Guides bleus ", 1927, p. 305.

36. MAîTre, Léon, Côtes bretonnes et vendéennes, de la Vilaine à Olonne. Guide du baigneur, Nantes, Imprimerie moderne, sans date (1895), p. 62.

37. Arch. dép. de Vendée, 4M 36, Saint-Jean-de-Monts. Arrêté municipal de Saint-Jeande-Monts du 27 juin 1850.

38. Arch. mun. de Saint-Gilles-Croix-de-Vie. Délibération municipale de Saint-Gilles-surVie du 19 novembre 1865.

39. Renaud, Henri, Guide illustré à Saint-Gilles-Croix-de-Vie et aux environs, Les Sablesd'Olonne, Roche-Jourdain imprimeur-éditeur, 3e éd. 1910, p. 194. 
dans son acception large, sept communes du marais en 662 mots tandis que les six communes du littoral dunaire (Saint-Gilles-sur-Vie et Croix-deVie n'ayant fusionné qu'en 1967) bénéficient d'un descriptif en 1518 mots $^{40}$. Saint-Hilaire-de-Riez est la seule commune où les deux versants du territoire sont réellement explorés. Localement, les agents promoteurs du tourisme ont désormais assimilé cette priorité. Dans le guide de l'Union des syndicats d'initiatives de Vendée, paru en 1935, la côte est largement évoquée tandis que le Marais breton est mentionné uniquement dans l'itinéraire $n^{\circ} 6$, " Environs de La Roche-sur-Yon-Le Marais Vendéen ", en quatre pages, les quatre dernières pages (soit $2 \%$ du volume de l'ouvrage) ${ }^{41}$. Dans les années 1950, les guides restent concentrés sur les stations balnéaires mais commencent à mentionner le marais, ses canaux, ses yoles et ses maraîchins.

Le professionnalisme des éditeurs des guides défavorise également les communes du Marais breton : plus aucune ville n'est désormais citée sans qu'un monument, qu'il soit naturel ou culturel, ne soit identifié. Or le Marais breton est réputé, à l'époque, pour ne pas disposer de patrimoine ancien exceptionnel et le patrimoine naturel local n'est pas encore reconnu. Conséquence de cet impératif, des communes sont désormais ignorées dans les guides : Bois-de-Céné, Châteauneuf, Le Fenouiller, Fresnay-en-Retz, Saint-Urbain sont oubliées; Notre-Dame-de-Riez, Le Perrier et Sallertaine sont citées une fois, Saint-Gervais deux fois. Ces communes, modestes en nombre d'habitants, sont également éloignées des plages où s'accumulent les touristes, alors que les voies de communication sont encore peu praticables quand le voyageur souhaite sortir du réseau départemental. Il ne faut pas oublier que, l'hiver venu, le marais est complètement inondé et que les voies de communication secondaires ne doivent pas contrarier la navigation en yole durant une partie de l'année.

Les autochtones commencent à revenir dans les discours touristiques à partir de la moitié du xx siècle, par petites touches. L'écrivain Jean Yole décrit les paysages et l'homme, dans ses occupations hivernales, quand " tout le marais alors lui appartient, et quand il passe sur sa yole, qu'il pousse avec élégance et adresse, son canardier à portée de sa main, il a l'air d'un gentilhomme chasseur ${ }^{42}$ ". Il évoque l'importance de l'élevage industriel, " de ces canetons challandais si réputés, dignes de finir, numérotés, à la Tour d'Argent ${ }^{43}$ ". La présence de l'habit ou du costume est parfois indiquée, comme la coiffe portée à Bourgneuf-en-Retz vers 1840, en forme de hennin très allongé ${ }^{44}$, ainsi que les danses maraîchines, qui ont lieu à chaque fête $^{45}$. La mention de la pêche aux anguilles dans le Marais, dispa-

40. MonmarchÉ, Georges, Bords de la Loire..., op. cit., 623 p.

41. Union des syndicats d'initiatives de Vendée, La Vendée touristique, 1935, 200 p.

42. YolE, Jean, "Vendée, terre de fidélité ", L'Opinion économique et financière, édition illustrée t. Iv Le Pays nantais et la Vendée, $\mathrm{n}^{\circ}$ 7, mars 1951, p. 82.

43. Ibidem, p. 83.

44. Syndicat d'initiatives régional de Nantes, Plages de la Loire-Atlantique, Angers, Éditions Jacques-Petit, 1951, p. 67.

45. Ibidem, p. 81. 
rue depuis cinquante ans, revient ${ }^{46}$. Une photo isolée, légendée «Bourrine et moulin en pays maraîchin ", montre bourrine, étier et moulin ailé, avec poteau électrique, dans le guide Rouge de 1957 mais l'auteur ne s'étend pas sur la région présentée comme " une contrée cultivée dominant le Marais vendéen sillonné de canaux ${ }^{47}$ ".

\section{De 1960 à nos jours : une prise de conscience de l'intérêt touristique du marais Breton}

L'édition de 1960 du guide Rouge Vendée, Charentes et plages, Poitou, Saintonge, Angoumois, présente une modification intéressante du discours touristique sur la région : elle comporte une introduction où les Marais, tant breton que poitevin, sont qualifiés de régions les plus pittoresques du Poitou $^{48}$. Cette introduction n'existait pas dans l'édition de 1957. Le Marais breton obtient une réelle identité : gastronomique, avec notamment le canard challandais mis en lumière lors du mariage du prince Rainier de Monaco et de Grace Kelly en 1956, au cours duquel il fut servi; paysagère, car l'espace est marqué par l'horizontalité et quelques points hauts, souvenirs des anciennes îles; emblématique, quand yole ou niole, selon la prononciation, et bourrine sont systématiquement évoquées; ethnographique, avec le maraîchinage. Le maraîchinage a été étudiée par l'érudit local Marcel Baudouin à la fin du XIX ${ }^{\mathrm{e}}$ siècle mais la première mention de cette pratique dans un guide date de 1965, dans le guide Michelin ${ }^{49}$, alors qu'elle n'existait plus dans les faits. Marcel Baudouin explique que la pratique du maraîchinage " consiste dans un accouplement bucco-lingual [prolongé], effectué, dans des conditions données, entre un jeune Maraîchin et une jeune Maraîchine, à l'âge où l'amour pousse dans le cerveau très neuf de nos alertes et vigoureux compatriotes, au moment où les sens s'éveillent ${ }^{50}$ ". L'ensemble de ces caractéristiques demeure exploité aujourd'hui : même le maraîchinage est encore cité dans le guide BaLaDO de $2013^{51}$. Cette période consacre donc un début de fixité de l'identité touristique du Marais breton.

Le Marais breton bénéficie d'une meilleure appréhension par les touristes grâce à l'ouverture du pays dans les années 1950 et 1960, ce qui bouleverse les traditions des habitants. Au même moment, des chercheurs, comme Claude Lévi-Strauss ou Edgar Morin, examinent la vie sociale rurale française, dans le village normand de Niels-Normandeux ${ }^{52}$ (1955)

46. Ibid.

47. Vendée, Charentes et plages, Poitou, Saintonge, Angoumois, Paris, Éditions BanetonThiolier, coll. " Guides Rouges ", 1957, p. 40 et 51.

48. Ibidem, 1960, p. 3-11.

49. Guide Michelin, Côte de l'Atlantique, de la Loire aux Pyrénées, Clermont-Ferrand,

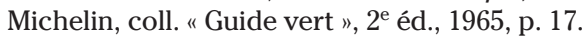

50. Baudouin, Marcel, Le maraîchinage, coutume du Pays de Monts, Saint-Grégoire, La Découvrance, retirage de l'édition de 1906 parue à Paris chez A. Maloine éditeur, p. 14 .

51. Guide BaLaDO, Pays de la Loire..., op. cit., p. 175.

52. MAUREL, Chloé, "La question des races ", Gradhiva [en ligne], 2007-5, mis en ligne le 12 juillet 2010, consulté le 5 février 2014. 
ou le village finistérien de Plozevet ${ }^{53}$ (1967). La vogue de l'ethnologie et de l'anthropologie en France, en grand renouveau dans les années 1960, impose aux guides la description de la population autochtone. Vers 1960, pour Armand de Baudry d'Asson, notable local, " le maraîchin est en effet d'esprit vif, curieux et subtil, tout en restant très indépendant. Il faut bien admettre que la vie moderne l'a pénétré, et ce n'est pas de sa faute s'il ne peut toujours profiter des facilités matérielles qu'elle procure ${ }^{54}$ ". Selon cet auteur, le maraîchin aperçoit d'ailleurs des étrangers venir dans son marais, à la recherche des planches permettant de franchir les fossés autrement qu'à la ningle. En 1964, Jean Debarre, instigateur du syndicat d'initiatives de Saint-Jean-de-Monts, décrit le maraîchin comme un personnage hors du commun : très vif, très intelligent, formant une race à part, au folklore intéressant, au parler qui attire les plus grands philologues du monde, où l'on retrouve des traces perdues ailleurs des vieux patois qui datent de plusieurs siècles ${ }^{55}$. En 1965, dans le guide Vert Michelin, le maraîchin, grand et fin, au teint coloré, se montre jaloux de son indépendance. Il reste traditionaliste dans le Marais vendéen, contrairement aux habitants du Marais poitevin, plus frondeurs ${ }^{56}$. En 1970, pour le comité départemental du tourisme, le maraîchin de Challans, différent physiquement et moralement des habitants des autres régions du marais, se montre fier d'allure; plus éleveur que cultivateur, musard à l'occasion, épris de grands rêves, excellent cavalier, mais peu résistant à la fatigue, hospitalier, très gai, aimant la musique et la danse, il est fidèle à la parole donnée, à ses convictions religieuses et à ses coutumes du bon vieux temps ${ }^{57}$. Malgré quelques critiques, le portrait apparaît élogieux, plus que ne l'était le portrait du XIX ${ }^{\mathrm{e}}$ siècle. Après cette décennie, à l'exception de la réédition du guide du Comité départemental du tourisme en 1974, le maraîchin n'est plus ethnographiquement décrit. La notion de race est critiquée, son usage est marginalisé, tandis que la pluralité et les relations interculturelles s'inscrivent dans le paysage intellectuel français ${ }^{58}$. Ce sont les pratiques et les objets qui sont dorénavant analysés.

Cette prise de conscience et la mutation du discours vont conduire les acteurs du tourisme et du patrimoine, dans les années 1980, à charmer les touristes avec des attractions ciblées dans le Marais. L'art de vivre, qui commençait à être évoqué depuis le milieu du $\mathrm{xx}^{\mathrm{e}}$ siècle, est systématiquement abordé, d'autant plus aisément que des structures muséales se

53. Morin, Edgar, Commune de France : la métamorphose de Plozévet, Paris, Fayard, $1967,287 \mathrm{p}$.

54. BAUDRY d'Asson, Armand de, " Marais breton ", dans Vendée : aspect géographique, historique, touristique, économique et administratif du département, Paris, Éditions Alépée et Cie, sans date (1960?), p. 40.

55. Saint-Jean-de-Monts, la plage moderne de l'Atlantique, Saint-Jean-de-Monts, 1964, p. 31 .

56. Guide Michelin, Côte de l'Atlantique..., op. cit., p. 17.

57. Comité départemental du tourisme, La Vendée, guide officiel, Toulouse, Éditions Larrieu-Bonnel, 1970, p. 29.

58. Liauzu, Claude, La société française face au racisme : de la Révolution à nos jours, Bruxelles, Éditions Complexe, coll. "Questions à l'histoire ", 1999, p. 156. 
mettent en place, dans les bourrines (habitat dont nous parlerons plus loin dans l'article) ou les exploitations fermières : moulin de Rairé (restauré en 1969), écomusée Le Daviaud (créé en 1982), bourrine du BoisJuquaud (rénovation en musée à partir de 1985), bourrine à Rosalie (transformation en site touristique entre 1971, date de la mort de la dernière habitante, Rosalie, et 1996, première mention dans un guide touristique). Ils tendent à se substituer au musée Bise-Dur, créé par Yvonne Cacaud et Marcel Baudouin, musée maraîchin situé dans le port de pêche de Croixde-Vie; aujourd'hui, c'est d'ailleurs devenu la Maison du pêcheur. En plus de mettre en représentation le Marais breton, ils invitent les touristes à se rendre hors des bourgs, contrairement aux autres musées de la région. Jusqu'alors, c'était les Maraîchins qui se dirigeaient vers les quartiers de la plage, comme dans les années 1920, quand la jeunesse montoise dansait la maraîchine au son de l'accordéon dans la cour de l'hôtel de la plage, à Saint-Jean-de-Monts ${ }^{59}$. Un discours associatif et professionnel s'agrège sur cet espace pour attirer les touristes venus dans les stations balnéaires voisines alors en état d'asphyxie ${ }^{60}$.

\section{Un Marais vivant et patrimonialisé?}

\section{Une construction du discours touristique en relation avec une construction identitaire}

La prise en compte du Marais par les auteurs des guides concorde avec des représentations festives réalisées par les habitants. Ainsi, une fête du Marais a lieu en 1952 à Bouin, peut-être en réaction à la fête organisée dans le Marais poitevin par l'Amicale maraîchine en juillet 1951, destinée à mettre en valeur le marais (groupe folklorique, bateaux fleuris, feu d'artifice) aux écluses de La Sotterie, dans les Deux-Sèvres ${ }^{61}$. Les estivants des localités voisines sont nombreux à s'être déplacés, friands de distractions. A lieu un défilé de chars et de groupes costumés, sans qu'on connaisse la thématique de ces déguisements ${ }^{62}$. À Saint-Gervais, lors de la grande kermesse du 27 juillet 1952, un char " La chasse dans le marais " est annoncé ${ }^{63}$. Les Maraîchins s'emparent donc de leur patrimoine et commencent à le faire connaître, avec succès puisque la présence des estivants est signalée. Cette présence extérieure est également un encouragement à la poursuite de la patrimonialisation. Il y a donc un basculement d'un savoir académique, dont Marcel Baudouin et sa sœur Yvonne Cacaud sont les dignes représentants au début du $\mathrm{xx}$ e siècle, vers un savoir plus populaire. Cette évolution a été observée ailleurs par Luc Noppen et Lucie K. Morisset mais,

59. Verdon, Henri, Le Pin [sic] est cuit : un touriste à Saint-Jean-de-Monts (1912-1980), Saint-Jean-de-Monts, APNS, 1981, p. 26-27.

60. Déjà en 1972, pour attirer les vacanciers dans le marais, Ouest-France titrait : «S’il y a trop de monde sur la plage" (édition du 21 juillet).

61. " Grande fête dans le Marais Poitevin ", Ouest-France du 26 juillet 1951.

62. "Bouin, La fête du marais ", Ouest-France du 10 juillet 1952.

63. "Saint-Gervais, grande kermesse ", Ouest-France du 21 juillet 1952. 
contrairement aux cas étudiés par ces deux auteurs ${ }^{64}$, l'Autre n'est pas ici l'intrus : il appuie la démarche, par sa présence. Certains syndicats d'initiatives locaux réalisent également des publications spécifiquement sur leur commune de rattachement, où le marais est mentionné sur quelques pages, même si la priorité reste la station balnéaire ${ }^{65}$. Il faut néanmoins remarquer qu'ils sont tous en bordure du marais, sur la côte ou dans les deux grandes villes, Challans et Machecoul, à la limite du bocage.

La phase de sauvegarde du patrimoine débute peu après. En 1961, OuestFrance interroge les syndicats d'initiatives vendéens pour connaître les améliorations possibles et permettre le développement touristique de la région. Chacun milite pour l'amélioration des voies de communication et de l'offre hôtelière (campings inclus). La période est donc encore dans la consolidation de la fréquentation touristique, avec l'apport du confort et la satisfaction des demandes en lien avec la hausse de l'affluence balnéaire. Toutefois, pour illustrer son papier, le journaliste place la photographie d'une bourrine, en interrogeant par cette légende ${ }^{66}:$ " Les bourrines vendéennes se font rares. A-t-on pensé à en conserver quelques-unes? " Dans son édition du 9 août, Ouest-France présente toujours ce dualisme : le texte du journaliste milite pour un meilleur confort et des dessertes routières facilitées tandis que l'illustration est une carte postale montrant des marấchins dans leur yole ${ }^{67}$.

\section{Une horizontalité paysagère atavique mais peu qualifiante}

Classiquement, les auteurs des guides optent pour le style narratif ou descriptif. Dans le cas présent, les principaux indicateurs langagiers découverts par Tropes sur l'ensemble de la période prouvent un style narratif : les verbes factifs en majorité (62,1\%), les modalisations de temps et de lieu, les connecteurs d'addition, d'opposition et de disjonction. Le locuteur expose une succession d'événements qui se déroulent à un moment donné, en un lieu donné. Le style descriptif opte pour des connecteurs de temps ( $2,4 \%$ du total) et de lieu ( 0$)$, quasiment absents, et des modalisations de manière, rares $(8,7 \% \text { du total })^{68}$. Ces caractéristiques sont similaires pour la période postérieure à 1960 , avec une légère progression pour les modalisations de manière $(9,7 \%$ du total). La description du marais est souvent brève. Vers 1960, elle se limite ainsi à : «Le Marais breton n'est pas

64. NOPPEN, Luc, et MORISSET, Lucie K., "Le patrimoine est-il soluble dans le tourisme? ", Téoros, vol. 22-3, 2003, mis en ligne le $1^{\mathrm{er}}$ avril 2012, consulté le 14 février 2014.

65. Saint-Jean-de-Monts, la plage moderne de l'Atlantique, Saint-Jean-de-Monts, 1964, p. 30, dans une partie "Les environs immédiats".

66. "La Vendée à l'heure touristique : communications, hôtellerie, grands soucis des syndicats d'initiatives ", Ouest-France du 19 juillet 1961.

67. "La Vendée à l'heure touristique : la "route bleue" sera-t-elle pittoresque ou utilitaire? ", Ouest-France du 9 août 1961.

68. WolfF, Marion, et VISSER, Willemien, " Méthodes et outils pour l'analyse des verbalisations : une contribution à l'analyse du modèle de l'interlocuteur dans la description d'itinéraires ", @ctivités, vol. 2, n 1, 2005, p. 165, revue en ligne, consulté le 14 février 2014. 
sensiblement différent du Marais poitevin; même faiblesse des altitudes, mêmes horizons illimités sous d'immenses ciels. Ici, les "îles" sont représentées par des émergences de calcaire grossier appartenant au début du tertiaire, telle la croupe de Sallertaine, à l'ouest de Challans ${ }^{69}$. " Ensuite sont égrenés les sites à visiter. À dix reprises dans le corpus, le nom commun " horizon " ou l'adjectif " horizontal " est utilisé, pour évidemment décrire un panorama mais aussi un dispositif d'architecture ou le mobilier présent dans les habitats jusqu'au XVIII" siècle. Le mot commun "plat ", en dehors de son sens gastronomique, n'a été relevé que trois fois. Les obstacles à cette horizontalité paysagère sont aussi rarement évoqués. Le logiciel Tropes relève seize occurrences " arbre " (une dix-septième occurrence porte sur la forêt domaniale), utilisées pour signifier leur absence ou leur rareté : "Aujourd'hui, le Marais breton, ce sont 36000 hectares d'un pays absolument plat où les rideaux d'arbres qui protègent les fermes constituent les seuls reliefs ${ }^{70}$. " Des collines sont tout de même signalées dix fois, mais trois de ces occurrences proviennent du recopiage d'une édition à l'autre du guide Joanne, entre 1886 et 1900. Elles matérialisent la limite des terres bocagères avec le marais. Le paysage du Marais breton n'est donc pas caractérisé par sa forme générale mais par ses composants.

Cette absence de site caché à l'œil n'empêche pas d'évoquer les " secrets" dont sont friands les guides. Le territoire acquiert cette dimension dans les années 1990, avec la progression de la reconnaissance du patrimoine naturel. La présence des hommes n'est jamais lointaine. Gilles Bély et Bertrand Bouflet promeuvent ainsi une promenade le long des étiers, de Soullans jusqu'au Perrier en passant par Sallertaine, " au cœur d'un pays toujours secret, fier de ses traditions [...] et heureux de les faire découvrir et partager ${ }^{71}$ ". Ce sont les habitants et les structures muséales qui transmettent les secrets, pas le guide. Nous retrouvons cette prise en compte de l'autochtone, en lien avec la patrimonialisation de la flore et de la faune, plus marquée à l'échelle planétaire à partir du congrès mondial des aires protégées de Durban en 2003. La position est moins naturaliste, elle devient plus sociale ${ }^{72}$. Le géographe Michel Picard concluait à l'inverse une décennie plus tôt ${ }^{73}$. L'évocation dans le texte de la faune ou de la flore se fait dans le cadre des musées ou des sentiers d'interprétations. Néanmoins, dans le même temps, grâce à une place de l'illustration plus

69. FÉnElon, Paul, "Le sol ", dans : Vendée : aspect géographique, historique, touristique, économique et administratif du département, Paris, Éditions Alépée et Cie, sans date (1960?), p. 15.

70. MARTin, Hubert, et Charrier, Thierry, 12 voyages en Pays de la Loire, Nantes, Siloë, 1999, p. 192.

71. BÉly, Gilles, et Bouflet, Bertrand, Vendée, tourisme..., op. cit., p. 193.

72. GLON, Éric, et CHEBANNE, Anderson, «Peuples autochtones et patrimonialisation de la nature protégée : les San indésirables dans le Central Kalahari (Botswana)? ", VertigO - la revue électronique en sciences de l'environnement, hors-série $n^{\circ} 16$, juin 2013 , mis en ligne le 30 mai 2013, consulté le 14 février 2014.

73. PICARD, Michel, Bali : tourisme culturel et culture touristique, Paris, L'Harmattan, 1992, p. 125. 
importante, la représentation de la faune et de la flore est devenue forte dans les guides; avec des restrictions d'usages liées à la protection du milieu naturel, la faune et la flore ne sont plus une ressource alimentaire et ludique (pêche), comme autrefois. La situation reste encore mouvante. Les guides ont fait infuser dans le discours touristique ce patrimoine naturel, devenu un spectacle permanent, à partir d'un inventaire d'une dizaine d'espèces : il est censé se retrouver partout ou presque. À quoi s'ajoutent quelques éléments culturels dispersés, comme la bourrine.

\section{Un habitat original tardivement reconnu : la bourrine}

La bourrine est l'un des éléments patrimoniaux majeurs du Marais breton, espace où l'habitat est de type isolé. Elle est mentionnée dans les guides dès 1829, par Eugène Genoude, dans la description d'un espace non pas typiquement de marais, mais une ancienne flèche dunaire qui s'enfonce désormais dans le marais, où a eu lieu le combat des Mathes : " de grands espaces sablonneux, d'anciens sillons remplis d'herbes, quelques arbres, quelques maisons blanches, très basses à cause des vents de mer, des bourrines, espèces de cahutes couvertes de jonc, des dunes dans le lointain ${ }^{74}$ ". Mais l'habitation s'avère en fait complètement ignorée par les guides à audience nationale, puisque la deuxième mention, chronologiquement, est portée dans le guide de Henri Renaud sur Saint-Gilles-Croixde-Vie et sa région, en $1910^{75}$, et la mention suivante figure dans le guide de l'Union des syndicats d'initiatives de Vendée, en $1935^{76}$. Les éditions régionales le représenteront dans la seconde moitié du $\mathrm{xx}^{\mathrm{e}}$ siècle. Le guide Rouge Vendée, Charentes et plages, Poitou, Saintonge, Angoumois, de 1957, y consacre une photo légendée ${ }^{77}$, et le guide Michelin Côte de l'Atlantique, de la Loire aux Pyrénées, de 1965, est le premier à la décrire en quelques mots : le Maraîchin "loge encore parfois dans des "bourrines", maisons basses, chaulées et couvertes de roseau ${ }^{78}$ ".

Cette mention tardive peut apparaître contradictoire avec l'évolution sociale que connaît le Marais breton. En 1968, l'érudit local Julien Rousseau reconnaît une transformation du Marais, où l'on peut encore trouver quelques bourrines échappées à la reconstruction ou à la modernisation et admirer la scrupuleuse propreté des intérieurs maraîchins, avec parfois les vieux meubles traditionnels (coffres de chêne cirés, armoires rustiques à fiches de cuivre, vaisseliers garnis de leurs faïences décorées, grands lits à quenouilles) : "Mais tout cela, depuis quelques lustres, c'est devenu quasi

74. GENOUDE, Eugène, Voyage dans la Vendée..., op. cit., p. 103-104.

75. Renaud, Henri, Guide illustré à Saint-Gilles-Croix-de-Vie et aux environs, Les Sables-

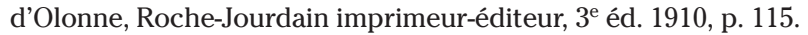

76. Union des syndicats d'initiatives de Vendée, La Vendée touristique, 1935, p. 17.

77. Vendée, Charentes et plages, Poitou, Saintonge, Angoumois, Paris, Éditions BanetonThiolier, coll. " Guides rouges ", 1957, p. 40.

78. Guide Michelin, op. cit., p. 17. 
des pièces de musée ${ }^{79} \ldots$. Jusqu'alors, la bourrine n'était pas considérée : habitation des plus pauvres du Marais, souvent installée sur les délaissés des chemins, sans aucun confort, elle comportait seulement une ou deux pièces $^{80}$. Les habitants préféraient, dès qu'ils en avaient la possibilité, faire construire un habitat moderne, laissant la bourrine se désagréger ou la détruisant de suite. Léon Dubreuil, dans l'introduction de son roman, Une enfance au Marais Breton (1930), affirme qu'il " est grand temps de les fixer [les mœurs maraîchines] avant qu'elles ne disparaissent comme les "bourines", les "nioles" et les pantalons à "godis" 81 ". La présence d'un char dénommé " La bourrine et le moulin " lors du défilé au moment de la Fête au Village de 1951, à Saint-Jean-de-Monts, montre une évolution ${ }^{82}$. Avec le début, entre 1960 et 1965, de l'achat par les touristes de bourrines, qu'ils font réhabiliter par les paysans ayant pris goût à la construction et au double salaire, la perception change radicalement.

La patrimonialisation s'empare en une vingtaine d'années de cet habitat original, dans un mouvement général de redécouverte de l'habitat traditionnel lié à l'élargissement, en 1964, du recensement des richesses monumentales à l'architecture rurale ${ }^{83}$ et, à partir des années 1970, au courant écologique. Ailleurs, la maison du Niou-Huella fait découvrir l'intérieur des maisons ouessantines dès 1968 et des opérations de réhabilitation de hameaux sont menées à partir de 1972 dans le parc des Cévennes ${ }^{84}$. Dans le Marais breton, les musées ouverts dans les années 1980 s'installent dans les bourrines délaissées par les héritiers des derniers occupants. La bourrine à Rosalie, musée privé, était occupée par une certaine Rosalie jusqu'en 1971, et l'écomusée de la bourrine du Bois-Juquaud s'installe dans le lieu où vécut jusqu'en 1970 Amandine Gaudemer, sans eau ni électricité, comme le firent ses ancêtres ${ }^{85}$. L'emblématisation ${ }^{86}$, au moment où la force symbolique de la bourrine fait l'objet d'un consensus communautaire, fige alors la représentation de l'habitat dans le Marais breton, alors que de nouveaux modèles architecturaux y font irruption et tout en délaissant les autres

79. Rousseau, Julien, À travers le Marais Breton Vendéen, Maulévrier, Éditions Hérault, $2^{\mathrm{e}}$ éd. 2001 [ $1^{\text {re }}$ éd. 1968], p. 135.

80. LE BEUF, François, " Maison de terre et de roseau : regards sur la bourrine du marais de Monts ", In Situ n ${ }^{\circ}$ 7, 2006, revue en ligne de l'Inventaire général, mis en ligne le 18 avril 2012, consulté le 14 février 2014.

81. DuBreult, Léon, Une enfance au Marais Breton, Auxerre, Imprimerie L'universelle, 1930, p. 10.

82. "À Saint-Jean-de-Monts, la Fête au Village ", Ouest-France du 3 septembre 1951.

83. Auduc, Arlette, "Paysage, architecture rurale, territoire : de la prise de conscience patrimoniale à la protection ", In Situ, n ${ }^{\circ}$ 7, 2006, revue en ligne de l'Inventaire général, mis en ligne le 11 avril 2012, consulté le 15 février 2014.

84. DAudé, Guy, "Une action du parc national des Cévennes : l'opération des hameaux ", Revue de géographie de Lyon, vol. 51, n², 1976, p. 177.

85. Guide BaLaDO, Pays de la Loire..., op. cit., p. 223, et Albertini, Sabine, Braconnier, Balthazar, Demeude, Hugues, Guitton, Pierre, Charente-Maritime, Vendée, Paris, GéoGuide, 2009 [1 $1^{\text {re }}$ éd. 2007], p. 436.

86. BOYER, Henri, De l'autre côté du discours : recherches sur le fonctionnement des représentations communautaires, Paris, L'Harmattan, 2003, p. 15-16. 
modes anciens d'habitat. Le guide touristique en matière de patrimoine rural n'apparaît pas avoir un rôle moteur dans le processus de patrimonialisation : il se limite à être une caisse de résonance des choix sociaux locaux sur cette question.

Le Marais breton est un espace rural dont l'appropriation touristique a été tardive. Le discours a donc d'abord évolué selon les intérêts des visiteurs qui logeaient en dehors de celui-ci. Dans un premier temps, l'attention s'est limitée à quelques éléments du patrimoine culturel mais ce territoire, comme beaucoup de zones rurales, se présentait comme un espace où il n'y avait rien de remarquable. Le Marais breton bénéficie en fait de la promotion interne d'une identité sur laquelle les guides vont pouvoir s'appuyer : une sorte de patrimonialisation interne mais qui n'a sans doute rien d'une génération spontanée. Les contextes local et national s'enrichissent : autochtones et visiteurs appuient mutuellement leurs démarches. Michel Picard, à partir de l'exemple de Bali, avait montré en 1992 que touristes et autochtones échangent mutuellement pour créer une culture satisfaisante pour l'ensemble des parties ${ }^{87}$. Nous aurions donc l'évolution inverse de ce qu'ont connu de nombreuses stations balnéaires françaises, peut-être du fait que le patrimoine est ici la conséquence d'un nouveau développement.

Le discours touristique est souvent présenté comme un discours qui s'enrichit, par sédimentation, mais notons que ce processus à travers les âges est imparfait : une partie des éléments anciens, promus dans les guides avant la Première Guerre mondiale, a disparu aujourd'hui des guides et des dépliants. La primauté de l'intérêt ne revient donc pas uniquement à l'ancienneté mais, aussi et surtout, à la reconnaissance locale. Quand Sophie Bonin constate, pour la description du fleuve Loire dans les guides Bleus entre 1856 et 1996, qu'elle se fait plus écologique au fil des décennies, avec une absence des sociétés locales, parce que le regard est devenu plus global, plus abstrait, optant pour une petite échelle ${ }^{88}$, nous devons constater que ce n'est pas tout à fait pareil pour le Marais breton. La description du patrimoine naturel revêt souvent une portée plus générale mais à cause de l'objet qu'il traite : l'auteur du guide peut difficilement promettre la présence permanente d'animaux à un endroit précis - c'est moins vrai pour les plantes. La présence des possibles médiateurs n'est, par contre, jamais oubliée. Il existe encore un entremêlement de la vie de l'homme avec ce milieu naturel particulier.

Dans le cas présent, la touristification se calque sur la patrimonialisation; mais pas entièrement. Le cas du maraîchinage est étonnant : cette pratique, perdue depuis des décennies, honnie par les élites locales au point que certains doutent de son existence ou de la réalité de sa description, présentée

87. PICARD, Michel, Bali.., op. cit., p. 196.

88. BonIN, Sophie, "Paysages et représentations dans les guides touristiques; La Loire dans la collection des Guides Joanne, Guides Bleus (de 1856 à nos jours) ", L'espace géographique, 2001-2, p. 118. 
comme fantasmée, perdure dans le discours touristique. Le discours se joue donc aussi des populations, en insérant des thématiques originales et, sans doute aussi pour cette raison, piquantes. S'il ne paraît pas encore exister de bréviaire pour "vendre " le tourisme rural, bien qu'il faudrait produire une étude poussée pour s'en assurer, il n'est pas exclu que les territoires ruraux soient bientôt perçus sous un nouveau filtre discursif.

\section{Annexe - Corpus des guides dépouillés}

Entre crochets, le lieu où l'exemplaire a été consulté ( $\mathrm{AD}$ = archives départementales; $\mathrm{AM}=$ archives municipales $; \mathrm{BM}$ = bibliothèque municipale)

Beauvoir-sur-Mer et sa région, Guide pratique France Magazine, 1990, 28 p. [AD Vendée].

De la Loire aux Pyrénées, Paris, Hachette et Cie, coll. " Guides Joanne ", 1911, 455 p. et nombreuses publicités. [BM Paris 16 $\mathrm{e}$ TCF].

Guide pittoresque du voyageur en France : département de la Loire-Inférieure, Nantes, Firmin Didot Frères, sans date (vers 1850), 24 p. [AD Loire-Atlantique].

Guide pittoresque du voyageur en France : département de la Vendée, Nantes, Firmin Didot Frères, sans date (vers 1850), 16 p. [AD Loire-Atlantique].

Guide de Nantes et des environs, Nantes, A. Dugas et Cie, 1909, 59 p. [AD Loire-Atlantique].

"Les petits trous pas chers "; Guide des familles aux bains de mer, plages de la Manche et de l'Océan, Paris, La Fare éditeur, 9e éd. 1903, 350 p. [BM Paris 16 ${ }^{\mathrm{e}} \mathrm{TCF}$ ].

L'Opinion économique et financière, édition illustrée t. IV Le Pays nantais et la Vendée, $\mathrm{n}^{\circ}$ 7, mars 1951, 104 p. [BM Paris 16 TCF].

Partons à la découverte de la côte atlantique, Levallois-Perret, Total Tourisme, 1975, 113 p. [BM Paris $\left.16^{\mathrm{e}} \mathrm{TCF}\right]$.

Saint-Jean-de-Monts, la plage moderne de l'Atlantique, Saint-Jean-de-Monts, 1964, 40 p. [AM Saint-Jean-de-Monts].

Saint-Jean-de-Monts, Guide officiel du Syndicat d'Initiative Office du Tourisme, SaintJean-de-Monts, 1971, 64 p. [AM Saint-Jean-de-Monts].

Saint-Jean-de-Monts, Guide officiel du Syndicat d'Initiative Office du Tourisme, SaintJean-de-Monts, 1974, 76 p. [AM Saint-Jean-de-Monts].

Saint-Jean-de-Monts, Guide officiel du Syndicat d'Initiative Office du Tourisme, SaintJean-de-Monts, 1975, 72 p. [AM Saint-Jean-de-Monts].

Vendée, Charentes et plages, Poitou, Saintonge, Angoumois, Paris, Éditions BanetonThiolier, coll. "Guides rouges ", 1957, 192 p. [BM Paris 16e TCF].

Vendée, Charentes et plages, Poitou, Saintonge, Angoumois, Paris, Éditions BanetonThiolier, coll. " Guides rouges ", 1960, 204 p. [BM Paris 16e TCF].

Vendée : aspect géographique, historique, touristique, économique et administratif du département, Paris, Éditions Alépée et Cie, sans date (1960?), 237 p. [BM Paris $\left.16^{\mathrm{e}} \mathrm{TCF}\right]$.

Guide Diamant, Nantes, Saint-Nazaire et les plages de la Loire-Inférieure, Paris, Hachette, 1921, 64 p. et de nombreuses publicités. [AD Loire-Atlantique].

Guide Michelin, Côte de l'Atlantique, de la Loire aux Pyrénées, Clermont-Ferrand, Michelin, coll. " Guide vert ", $2^{\mathrm{e}}$ éd., 1965, 162 p. [BM Paris 16 TCF].

Guide Gallimard, Nantes-Côte de Jade, Paris, Éditions Nouveaux-Loisirs, 1992, 396 p. [BM Saint-Hilaire-de-Riez]. 
Guide Gallimard, La Vendée maritime, Paris, Éditions Nouveaux-Loisirs, 1994, 322 p. [BM Saint-Hilaire-de-Riez].

Guide Bleu, Pays de la Loire, Paris, Hachette, 1995 [1 $1^{\text {re }}$ éd. 1989], 767 p. [BM SaintHilaire-de-Riez].

Guide Vert Michelin, Poitou, Vendée, Charentes, Clermont-Ferrand, Michelin, 1996, 300 p. [BU Saint-Hilaire-de-Riez].

Guide du Routard, Pays de la Loire, Paris, Hachette, 2007, 560 p. [BM Saint-Hilairede-Riez].

Guide BaLaDO, Pays de la Loire, Paris, Éditions Mondéos, 2013, 476 p. [BM SaintJean-de-Monts].

Albertini, Sabine, Braconnier, Balthazar, Demeude, Hugues, Guitton, Pierre, CharenteMaritime, Vendée, Paris, GéoGuide, 2009 [1 $1^{\text {re }}$ éd. 2007], 512 p. [BM Saint-Hilairede-Riez].

ARDouin-Dumazet, Voyage en France t. II, Paris/Nancy, Berger-Levault et Cie, 1894, 334 p. [AD Loire-Atlantique].

Ardouin-Dumazet, Voyage en France : $3^{e}$ série, les îles de l'Atlantique, Paris/Nancy, Berger-Levrault et Cie, 1895, 314 p. [BM Paris 16e TCF].

BÆDEKER, K., Le Sud-Ouest de la France, de la Loire à la frontière d'Espagne : manuel du voyageur, Leipzig/Paris, Bædeker/Ollendorff, $6^{\mathrm{e}}$ éd., 1897, 384 p. et deux cartes. [BM Paris $16^{\mathrm{e}} \mathrm{TCF}$.

BÆDEKER, K., Le Sud-Ouest de la France, de la Loire à la frontière d'Espagne : manuel du voyageur, Leipzig/Paris, Bædeker/Ollendorff, 9éd., 1912, 512 p. et deux cartes. [BM Paris 16 TCF].

BÉLY, Gilles, et Bouflet, Bertrand, Vendée, tourisme et culture, La Roche-sur-Yon, Siloë éditeur, coll. " Guides Siloë ", 2001, 272 p. [BM Saint-Hilaire-de-Riez].

Comité départemental du Tourisme, La Vendée, guide officiel, Toulouse, Éditions Larrieu-Bonnel, 1970, 224 p. [AD Vendée].

BROCHET, Louis, La Vendée pittoresque, historique et archéologique : guide du touriste, Fontenay-le-Comte, Henri Lussaud imprimeur-éditeur, 1921, 144 p. [BM Paris $\left.16^{\mathrm{e}} \mathrm{TCF}\right]$.

BRoquelet, A., À travers nos provinces : de la Vendée aux Pyrénées-Orientales, Paris, Garnier, 1930, 310 p. [BM Paris 16 $\mathrm{TCF}$ ].

Comité départemental du tourisme de Loire-Atlantique, Promenades et randonnées en Loire-Atlantique, Rennes, Éditions Ouest-France, 1993, 143 p. [AD Loire-Atlantique].

Comité départemental du tourisme, La Vendée, guide officiel, Toulouse, Éditions Larrieu-Bonnel, 1970, 224 p. [BM Paris $16^{\mathrm{e}} \mathrm{TCF}$ ].

Comité départemental du tourisme de Vendée, La Vendée, Toulouse, Éditions Larrieu-Bonnel, 1974, 230 p. [AD Vendée].

Comité départemental du tourisme de Vendée, La Vendée autrement, La Roche-surYon, 1986, non paginé. [AD Vendée].

Comité régional du tourisme des Pays-de-la-Loire, Guide Loisirs Accueil : Pays de la Loire, Paris, Guides Loisirs Accueil, 1983, 326 p. [AD Loire-Atlantique].

Conseil général de Vendée, Vendée randonnées : guide avec cartes détaillées, La Roche-sur-Yon, sans date (années 2000), 256 p. [BM Saint-Jean-de-Monts].

Genoude, Eugène, Voyage dans la Vendée et dans le Midi de la France, Paris/Lyon, Méquignon Fils aîné/Périsse Frères, 1829, 420 p. [BM Paris 16e TCF].

GruYEr, Paul, Un mois en Bretagne, Paris, Hachette, 1925, 224 p. [AD Loire-Atlantique].

JoAnne, Adolphe, Géographie du département de la Vendée, Paris, Hachette et Cie, 1900 [reprint 1994], 64 p. et carte de la Vendée [BM Saint-Jean-de-Monts].

LE LANNou, Maurice, Itinéraires de Bretagne : guide géographique et touristique, Paris, 
Baillière et Fils éditeurs, sans date (années 1930), 296 p. [AD Loire-Atlantique].

MAITTRE, Léon, Côtes bretonnes et vendéennes, de la Vilaine à Olonne; Guide du baigneur, Nantes, Imprimerie moderne, sans date (1895), 80 p. [AD Loire-Atlantique].

Martin, Hubert et Charrier, Thierry, 12 voyages en Pays de la Loire, Nantes, Siloë, 1999, 224 p. [AM La Roche-sur-Yon].

MonmarchÉ, Georges, Bords de la Loire et Sud-Ouest, Paris, Hachette, coll. « Guides bleus ", 1927, 623 p. [BM Paris 16 $\mathrm{e}$ TCF].

RIvÉ, Pierre, Le guide de la Vendée, Lyon, La Manufacture, 1988, 206 p. [AM La Rochesur-Yon].

Syndicat d'initiatives régional de Nantes, Plages de la Loire-Atlantique, Angers, Éditions Jacques-Petit, 1951, 136 p. [BM Paris 16 TCF].

Union des syndicats d'initiatives de Vendée, La Vendée touristique, 1935, 200 p. [AD Loire-Atlantique].

\section{RÉSUMÉ}

Aux limites de stations balnéaires de réputation nationale, le marais Breton, situé à la frontière des départements de la Vendée et de la LoireAtlantique, n'a pourtant pas été considéré, pendant longtemps, comme un espace touristique potentiel. Face à la difficulté d'appréhender ce territoire, les guides ont progressivement forgé leurs discours, dont on peut voir la progression à travers un corpus de 48 ouvrages : de la méfiance au XIX ${ }^{\mathrm{e}}$ siècle à la découverte d'un patrimoine rural et naturel original, dans la seconde moitié du $\mathrm{Xx}^{\mathrm{e}}$ siècle. Ce processus, de plus d'un siècle, toujours vivace, s'appuie sur une patrimonialisation qui est moins le fait des érudits locaux que de la population qui s'en empare, non sans contradictions. Cet entremêlement de la vie de l'homme avec ce milieu naturel particulier y apparaît essentiel. Dans cet espace rural, on peut donc analyser une touristification qui se développe en même temps qu'une patrimonialisation.

\section{ABSTRACT}

The Breton marsh, despite being situated near some busy seaside resorts, was for a long time not considered as a potential tourist area. Authors of guidebooks found this area difficult to incorporate as it was divided between two French Departments. The analysis of 48 guidebooks shows how the Breton marsh was described: the discourse evolved from being wary in the nineteenth century, to celebrating the rediscovery of its unique natural and rural heritage in the second half of the twentieth century. This long process of transformation was more the result of the support of the local population than of the knowledge of local scholars. This mix of tourism and of heritage recognition took place simultaneously. In order to explain this phenomenon, this study analyses the interaction between social life and the natural environment. 